Pictured right is a Mach-Zehnder interferometer, one of several proposed switching devices ${ }^{7}$. Light is guided through a 1-micrometre channel between walls of slightly lower refractive index. The beam is split between two legs one of which has two electrodes above and below. Applying a voltage changes the effective refractive index in that channel so that the light slows until it is exactly out of phase with the other leg: when the beams are recombined, nothing emerges. This can convert electronic data to light pulses for transmission between parts of a computer that are too far apart for electronic conduction to occur rapidly. A similar logic device could have one leg intrinsically nonlinear such that the light in that leg slows as the intensity increases until it becomes out of phase with the other leg. This could then be part of an all-optical computer. The small channel size allows very high light intensities to be reached with relatively low total powers.

signal, but it is also intractable and unstable. As $\chi^{(3)}$ increases with the extent of conjugation to the fifth power, long conjugated chains should have huge values. Unfortunately these systems also absorb light.

The polydiacetylenes are coloured but transparent at long wavelengths and some can be cast as films from solution. They can also be prepared as LangmuirBlodgett films (similar to soap films), but these tend to include light-scattering defects and the optically active groups are diluted by the soluble hydrocarbon tails needed at the processing stage.

Polybenzbisthiazole (PBT) also has a $\chi^{(3)}$ of around $10^{-10}$ e.s.u. (ref.3; Prasad, State University of New York, Buffalo). This is one of a group of rigid rod polymers developed from work at Wright Patterson Air Force Base on high-temperature polymers. Polyphenylenevinylene is another black, conjugated, conducting polymer and has $\chi^{(3)}=3 \times 10^{-10}$ e.s.u. when drawn into an oriented film.

The polysilanes, based on a silicon backbone rather than carbon, are an interesting possibility ${ }^{4.5}$. For polymethylphenylsilane $\chi^{(3)}=10^{-12}$ e.s.u. when measured with 1.06-micrometre light and decreases at longer wavelengths as the third harmonic is enhanced by resonance. Polysilanes are clear and can be easily made as films. They would be expected to have just a $\sigma$-bond system similar to polyethylene and no significant nonlinearity. The effect seems to arise from delocalization of electrons along the silicon-chain backbone which allows enough 'softness' to respond to the optical input. There is some evidence for this delocalization in the optical spectrum ${ }^{6}$. Also new data show that the response is very fast (taking less than 3 picoseconds) and is therefore due to electronic motion rather than molecular motion or thermal effects ${ }^{5}$.

One expects the nonlinearity of an organ pipe to increase as the resonant frequency is approached and it starts to shake vigorously. In molecules resonance is usually associated with absorption and so is to be avoided. If the aim is to generate third-harmonic light, there should be no

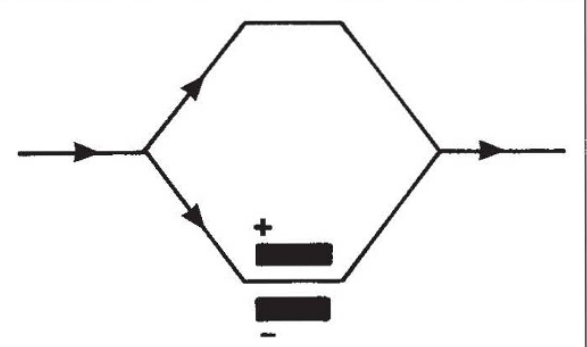

absorption at either the fundamental frequency or at the third harmonic. But to obtain a simple change of refractive index absorption at the third harmonic can enhance $\chi^{(3)}$ without detracting from the transmission of the fundamental. In general the measured $\chi^{(3)}$ rises as the fundamental wavelength is reduced until the third harmonic approaches an absorption band. In other words, absorption seems to be the price of a high $\chi^{(3)}$. Much attention was given at the meeting to molecular orbital calculations of $\chi^{(3)}$. which now can get within an order of magnitude of the right answer. We may be approaching a point where some useful prediction can be made of good structures.

Large inorganic crystals are already used for frequency doubling and tripling for laser spectroscopy, but these materials are difficult to adapt to small waveguides. Organic materials with extended conjugation offer large nonlinear coefficients and rapid switching when compared to inorganic materials. Polymers can be stable, easily formable and can (with difficulty) be made to high optical transparency. Whether these properties can all be combined in a single system remains to be seen. One view is that the stringent requirements of purity and stability in a computing system will prove to be too much for organics, as seems to be the case with conducting polymers. Another view is that opto-electronic transducers have been functioning effectively in chloroplasts and retinas for a long time and there should be no problem.

1. Sinclair, M., Moses, D., Akagi, K. \& Heeger, A.J. in MRS Symp. 109, 205-216 (Materials Research Society, Pittsburgh. 1988).

2. Kajzar. F. \& Messier. J. in Nonlinear Optical Properties of Organic Molecules and Crystals Vol. 2 (eds Chemla, D.S. \&

Zyss, J.) 51-84 (Academic, Orlando, 1987).
Garito, A. \& Teng, C. C. SPIE Proc. 613, 146-152 (1986).

4. Kajzar, F.. Messier, J. \& Rosilio, C. J. appl. Phys. 60, 3040 3044 (1986)

5. Yang, L. et al. Appl. Phys. Lett. 53, 1245-1247 (1988).

6. Harrah, L. \& \%eigler, J.M. Macromolecules 20,601-608 (1987)

7. Stegeman, G.I. Zanoni, R. \& Seaton. C.T. in MRS Symp. 109. 53-64 (Materials Research Society, Pittsburgh. 1988).

Paul Calvert is in the Department of Materials Science and Engineering, University of Arizona, Tuscon, Arizona 85712, USA.

\section{Nature statistics}

IN 1988, about 6,000 unsolicited manuscripts were submitted to Nature for publication as Letters or Articles - almost exactly the same number as in 1987 . About 35 per cent were sent to our Washington office rather than to London - there is no preference. About 30 per cent dealt with physical sciences rather than biological sciences. As usual, submissions peaked in the summer months to coincide with the period when potential referees (and Nature staff) are most likely to be away.

Of the submitted papers, just over 1,000 were published, 400 in the physical sciences and 600 in the biological sciences. If 1988 is no exception to the pattern of the past few years documented by the Science Citation Index, 30 of the 600 biological papers will be among the 100 life-science papers published in 1988 most cited by the end of 1989 .

On the average, the delay between receipt and the appearance in print of the papers accepted for publication was just over 4 months. (Papers not accepted for publication are returned to their authors much more quickly.) As the figure shows, 30 or so papers, mostly on high-temperature

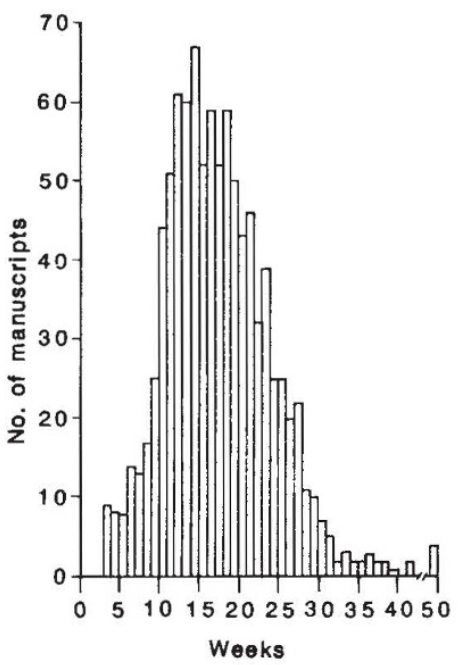

superconductivity, were published within 6 weeks of submission, but an equal number took more than 8 months to appear in print - sometimes because they were overgenerously granted the original date of submission despite long delays during substantial revision. The median time between receipt and acceptance was 10 weeks, and from acceptance to publication was 7 weeks.

Naturally, we hope that publication will be even faster in 1989. One helpful development is the increasing use being made of telefax machines. For example, the proportion of referees' reports sent to our Washington office by that means increased from 8 per cent in June 1988 to 35 per cent in December. During 1989, we expect a further increase in the use of fax machines and some growth in the use of electronic mail to deliver reviews of manuscripts. 